\section{Análisis de los fenotipos y genotipos de resistencia a eritromicina y clindamicina en cepas de Streptococcus pyogenes aisladas en Chile en un período de 10 años}

\author{
CARLOS RODRÍGUEZ ${ }^{1, \mathrm{a}}$, PABLO ROJAS ${ }^{1, \mathrm{a}}$, ANIELA WOZNIAK ${ }^{1, \mathrm{~b}}$, \\ ALEXIS M. KALERGIS ${ }^{2,3, c}$, INÉS CERÓN ${ }^{4}$, INGRID RIEDEL ${ }^{1, d}$, \\ JUAN C. ROMÁN ${ }^{1, d}$, LUIS A. VILLARROEL ${ }^{5, \mathrm{e}}$, XIMENA BERRÍOS ${ }^{5}$, \\ LUIS BAVESTRELLO ${ }^{6}$, PATRICIA GARCÍA ${ }^{1}$
}

\section{Resistance phenotypes and genotypes of Streptococcus pyogenes clinical isolates in Chile over a 10 -year period}

Background: Macrolide and lincosamide resistance in Streptococcus pyogenes is due to the acquisition of mef, ermB and ermA genes, which confer different resistance phenotypes, namely M, MLSBconstitutive and MLSBinducible respectively. The last report of resistance in Chile was done in the period 1990-1998, in which resistance to macrolides was 5.4\%, with M phenotype as the predominant one. Aim: To characterize the evolution of erythromycin and clindamycin resistance and their associated genes in $S$. pyogenes strains isolated from patients with invasive and noninvasive infections in the period 1996 to 2005. Material and Methods: Resistance to erythromycin and clindamycin was determined in 1,282 clinical isolates using the disk diffusion test. Resistant isolates were analyzed by polymerase chain reaction (PCR) for the presence of the above mentioned resistance genes. Results: Global resistance to erythromycin and clindamycin was 3.5 and $0.7 \%$ respectively. Eighty percent of the resistant strains possessed the $M$. phenotype. Conclusions: Resistance levels of $S$. pyogenes have decreased in Chile in the last years. Most resistant strains have $M$ phenotype in contrast to many countries in which the MLSB constitutive phenotype is the predominant one.

(Rev Med Chile 2011; 139: 1143-1149).

Key words: Drug resistance, bacterial; Genetic markers; Streptococcus pyogenes.

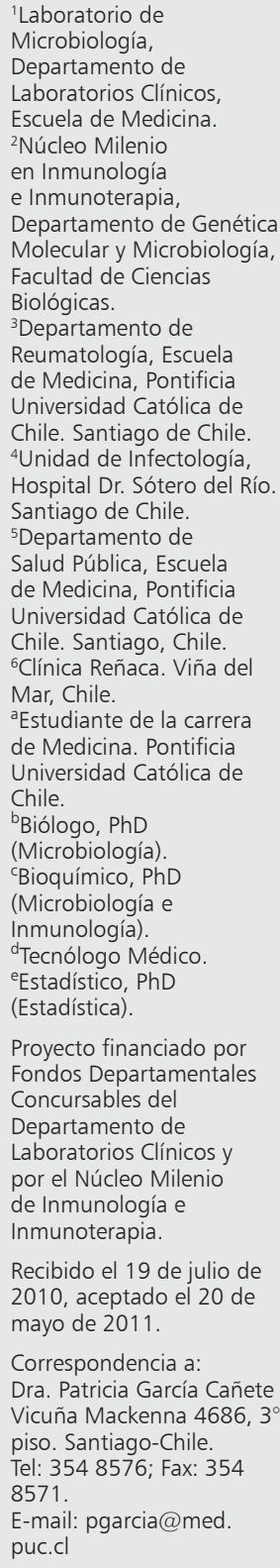

Si treptococcus pyogenes es una bacteria Grampositiva, $\beta$-hemolítica perteneciente al grupo A de Lancefield. Es responsable de infecciones no invasoras como amigdalitis, escarlatina y afecciones de la piel ${ }^{1} \mathrm{y}$ de infecciones invasoras graves como bacteriemia ${ }^{2}$, fasceítis necrotizan$\mathrm{te}^{3,4} \mathrm{y}$ shock séptico ${ }^{5}$. Adicionalmente, $S$. pyogenes produce patologías con secuelas, siendo las más importantes la fiebre reumática y la glomerulonefritis post-infecciosa ${ }^{6}$.

A comienzos del siglo XX hubo grandes brotes de infecciones con alta mortalidad ${ }^{6}$. Con el descubrimiento de la penicilina se logró controlar la enfermedad ${ }^{7}$. Sin embargo, en las décadas de198089 y 1990-99 ocurrió una reaparición mundial de casos de fiebre reumática y de infecciones 
sistémicas con complicaciones severas ${ }^{8,9}$. En Chile, durante la década de 1980-89 hubo un brote de glomerulonefritis aguda post-estreptocócica ${ }^{10}$ y en 1999 Abarca y cols reportaron un brote de infecciones invasoras (comunicado en el XVI Congreso Chileno de Infectología, 1999). Este cambio epidemiológico ha motivado nuevos estudios ${ }^{11}$.

El antibiótico de elección para el tratamiento de infecciones por $S$. pyogenes es la penicilina, indicándose los macrólidos y lincosamidas en los pacientes alérgicos a antibióticos $\beta$-lactámicos. Adicionalmente, en infecciones invasoras se administra clindamicina pues la monoterapia con penicilina no es absolutamente eficaz ${ }^{12}$. En estos casos la resistencia a macrólidos y lincosamidas empeora el pronóstico de las infecciones. Por esta razón el estudio y vigilancia de la resistencia en $S$. pyogenes es de gran importancia.

Varios factores influyen en el surgimiento de cepas resistentes, pero la presión selectiva ejercida por el uso indiscriminado de antibióticos es uno de los más importantes ${ }^{13}$. Se ha reportado una correlación entre el consumo de antibióticos y resistencia de Streptococcus spp en infecciones extrahospitalarias ${ }^{14,15}$, fenómeno corroborado en varios países ${ }^{16}$.

Los macrólidos y las lincosamidas inhiben la síntesis proteica por unión al ARN ribosomal $23 \mathrm{~S}^{17}$. S. pyogenes adquirió resistencia a estos antibióticos por dos mecanismos. El primero, por adquisición de un gen que codifica para una metilasa del ARN ribosomal 23S bloqueando la unión del antibiótico ${ }^{18}$. S. pyogenes posee la metilasa ErmB (gen ermB) la cual confiere resistencia constitutiva a macrólidos, lincosamidas y estreptogramina B conocido como fenotipo MLS $_{B}$ constitutivo $\left(\mathrm{MLS}_{\mathrm{B}} \mathrm{const}\right)^{19}$. S. pyogenes también puede poseer la metilasa ErmA codificada por el gen erm ${ }^{20}$. Las cepas portadoras de esta metilasa poseen resistencia constitutiva a macrólidos e inducible a lincosamidas (fenotipo MLS ${ }_{\mathrm{B}}$ inducible, $\mathrm{MLS}_{\mathrm{B}}$ ind $)^{21}$. El segundo mecanismo de resistencia a macrólidos consiste en la adquisición de un gen llamado mef que codifica para una bomba de expulsión que transporta los macrólidos fuera de la célula bacteriana. Las cepas que lo adquieren son resistentes solamente a macrólidos y poseen el fenotipo $\mathrm{M}^{22,23}$.

En Chile, Berríos y cols realizaron un estudio en muestras de $S$. pyogenes obtenidas entre 1982 y 1987 donde no se encontraron cepas resistentes a eritromicina ${ }^{24}$. Las primeras cepas resistentes a eritromicina en Chile fueron descritas en 1994 y a clindamicina en $1995^{25}$. Posteriormente, Palavecino y $\operatorname{cols}^{26}$ determinaron los niveles de resistencia en Chile entre los años 1990 y 1998 y describieron por primera vez en el país los genotipos asociados. Sin embargo, a partir de 1999 se desconocen los niveles de resistencia así como los genotipos asociados. El objetivo de nuestro trabajo fue caracterizar la evolución de la resistencia a eritromicina y clindamicina y sus genes asociados en cepas de $S$. pyogenes aisladas en Chile de pacientes con infecciones invasoras y faríngeas (no invasoras) entre los años 1996 y 2005.

\section{Material y Método}

\section{Cepas bacterianas}

Para conocer la evolución de la resistencia, se seleccionaron todas las cepas de Streptococcus pyogenes aisladas desde 1996 hasta 2005 en el Laboratorio de Microbiología del Servicio de Laboratorios Clínicos de la Pontificia Universidad Católica de Chile, las cuales hacían un total de 1.282 cepas. De éstas, 108 eran cepas invasoras, es decir, aisladas de sitios estériles. Las 1.174 cepas restantes fueron aisladas de exudados faríngeos. Todas las cepas de exudado faríngeo provienen de pacientes de la Región Metropolitana (1.174) y sólo 32 cepas invasoras son representativas de otras regiones.

\section{Ensayo de susceptibilidad a agentes antimicrobianos}

La susceptibilidad a antibióticos fue determinada para todas las cepas estudiadas mediante el método de difusión en agar, siguiendo los lineamientos del Instituto para la Estandarización de Laboratorios Clínicos (CLSI) ${ }^{27}$. Los antimicrobianos ensayados fueron penicilina, eritromicina, clindamicina, cefotaxima y vancomicina. El control de calidad del ensayo se realizó con la cepa de S. pneumoniae ATCC 49619. Para determinar el fenotipo de resistencia se utilizó el test del doble disco (test D). El mismo consiste en situar el disco de eritromicina a $16 \mathrm{~mm}$ del disco de clindamicina (de borde a borde). El aplanamiento del halo de inhibición de la clindamicina en la cercanía del disco de eritromicina se interpreta como fenotipo MLS $_{B}$ ind. Si es resistente a eritromicina y susceptible a clindamicina sin aplanamiento del halo se 
interpreta como fenotipo M. Aquellas cepas resistentes a eritromicina y a clindamicina se interpretaron como fenotipo $\mathrm{MLS}_{\mathrm{B}}$ const. Los diámetros de los halos fueron interpretados según los criterios del CLSI ${ }^{27}$.

\section{Amplificación de los genes de resistencia a antibióticos}

Se extrajo el ADN de todas las cepas resistentes utilizando el kit para extracción de ADN genómico QuiAmp (Qiagen). Además se extrajo el ADN de 2 cepas sensibles para ser usadas como control negativo. Tres cepas de $S$. pyogenes portadoras de cada uno de los genes mef, ermB y ermA, respectivamente, fueron utilizadas como controles positivos. El ADN de cada una de las cepas resistentes fue amplificado por PCR con los siguientes partidores específicos para cada uno de los 3 genes de resistencia: ermB, mef y ermA: ermB fwd: 5'-CGAGTGAAAAAGTACTCAACC-3'y rev: 5'-GGCGTGTTTCATTGC TTGATG-3', ermA fwd: 5'-GCATGACATAAACCTTCA-3' y rev: 5'-AGGTTATAATGAAACAGA-3', mef fwd: 5'-AGTATCATTAATCACTAGTGC-3' y rev: 5'-TTCTTCTGGTACTAAAAGTGG-3'. Las reacciones de PCR fueron realizadas de acuerdo a los protocolos ya descritos ${ }^{28,29}$. Los productos de PCR fueron visualizados bajo luz u.v. mediante electroforesis en geles de agarosa al 1\% teñidos con bromuro de etidio.

\section{Análisis estadístico}

Se utilizó test $\chi^{2}$ o test exacto de Fisher para la comparación de porcentajes de resistencia en cepas invasoras y faríngeas. Se utilizaron modelos de regresión Poisson para analizar la tendencia de las tasas de resistencia en el tiempo. Se utilizó la regresión joinpoint para determinar si existían cambios de tendencia en las tasas de resistencia en el tiempo ${ }^{30}$.

Se consideró significativo todo valor $\mathrm{p}$ inferior o igual a 0,05. Para los análisis se usó el programa estadístico $\mathrm{R}^{31}$.

\section{Resultados}

Determinación del fenotipo de resistencia a antibióticos

El estudio fenotípico de resistencia en las 1.282 cepas aisladas de pacientes con infec- ciones invasoras (108 cepas) y faríngeas ( 1.174 cepas) mostró que el $100 \%$ de las cepas era sensible a penicilina, cefotaxima y vancomicina. Sin embargo, se observó que $3,5 \%$ de las cepas ( 45 cepas) del período estudiado eran resistentes a eritromicina (Tabla 1). De las 45 cepas resistentes a eritromicina, 9 de ellas eran además resistentes a clindamicina, lo que hace un porcentaje global de resistencia a este antibiótico de 0,7\% (Tabla 1). Respecto a la evolución del número de cepas resistentes a eritromicina se observa que el porcentaje de resistencia en el año 1997 fue de alrededor de $10 \%$ y en 1998 fue de 6,7\%, mientras que luego del año 1999 los valores eran en su gran mayoría menores a $4 \%$ (Tabla 1). Para analizar estadísticamente esta observación, se ajustó un modelo de regresión Poisson para analizar la tendencia en función del tiempo. Existe una tendencia decreciente y significativa con valor $\mathrm{p}<0,001$. No se observaron cambios o quiebres en la tendencia, según resultados obtenidos utilizando la regresión joinpoint $t^{30}$. Estos resultados indican una disminución en el surgimiento de cepas resistentes en Chile en los últimos años del estudio.

El análisis de la resistencia de cepas invasoras demostró una resistencia a eritromicina de 3,7\% y de $0,9 \%$ a clindamicina, mientras que las cepas faríngeas presentaron una resistencia a eritromicina de 3,5\% y

Tabla 1. Evolución de resistencia a eritromicina y clindamicina en aislados clínicos entre 1996-2005 en Chile

\begin{tabular}{|lccc|}
\hline Año & $\begin{array}{c}\mathbf{N}^{\circ} \text { de cepas } \\
\text { estudiadas }\end{array}$ & \multicolumn{2}{c|}{$\mathbf{N}^{\circ}$ de cepas resistentes a: } \\
Eritromicina* & Clindamicina* \\
\hline 1996 & 21 & $1(4,7 \%)$ & $0(0 \%)$ \\
\hline 1997 & 61 & $6(9,8 \%)$ & $1(1,7 \%)$ \\
\hline 1998 & 60 & $4(6,7 \%)$ & $1(1,7 \%)$ \\
\hline 1999 & 144 & $1(0,7 \%)$ & $0(0 \%)$ \\
\hline 2000 & 109 & $6(5,5 \%)$ & $0(0 \%)$ \\
\hline 2001 & 146 & $7(4,8 \%)$ & $1(0,7 \%)$ \\
\hline 2002 & 203 & $3(1,4 \%)$ & $0(0 \%)$ \\
\hline 2003 & 236 & $8(3,4 \%)$ & $1(0,4 \%)$ \\
\hline 2004 & 161 & $2(1,2 \%)$ & $0(0 \%)$ \\
\hline 2005 & 141 & $7(5,0 \%)$ & $5(3,5 \%)$ \\
\hline Total & 1.282 & $45(3,5 \%)$ & $9(0,7 \%)$ \\
\hline
\end{tabular}

*El porcentaje correspondiente a cada valor se muestra entre paréntesis. 
Tabla 2. Distribución de la resistencia entre las cepas invasoras y faríngeas estudiadas en el período 1996-2005

\begin{tabular}{|c|c|c|c|}
\hline & \multirow{2}{*}{$\begin{array}{c}\mathbf{N}^{\circ} \text { cepas } \\
\text { estudiadas }\end{array}$} & \multicolumn{2}{|c|}{$\mathbf{N}^{\circ}$ cepas resistentes a: } \\
\hline & & Eritromicina* & Clindamicina* \\
\hline Invasoras & 108 & $4(3,7 \%)$ & $1(0,9 \%)$ \\
\hline Faríngeas & 1.174 & $41(3,5 \%)$ & $8(0,7 \%)$ \\
\hline Total & 1.282 & 45 & 9 \\
\hline
\end{tabular}

* El porcentaje correspondiente a cada valor se muestra entre paréntesis.
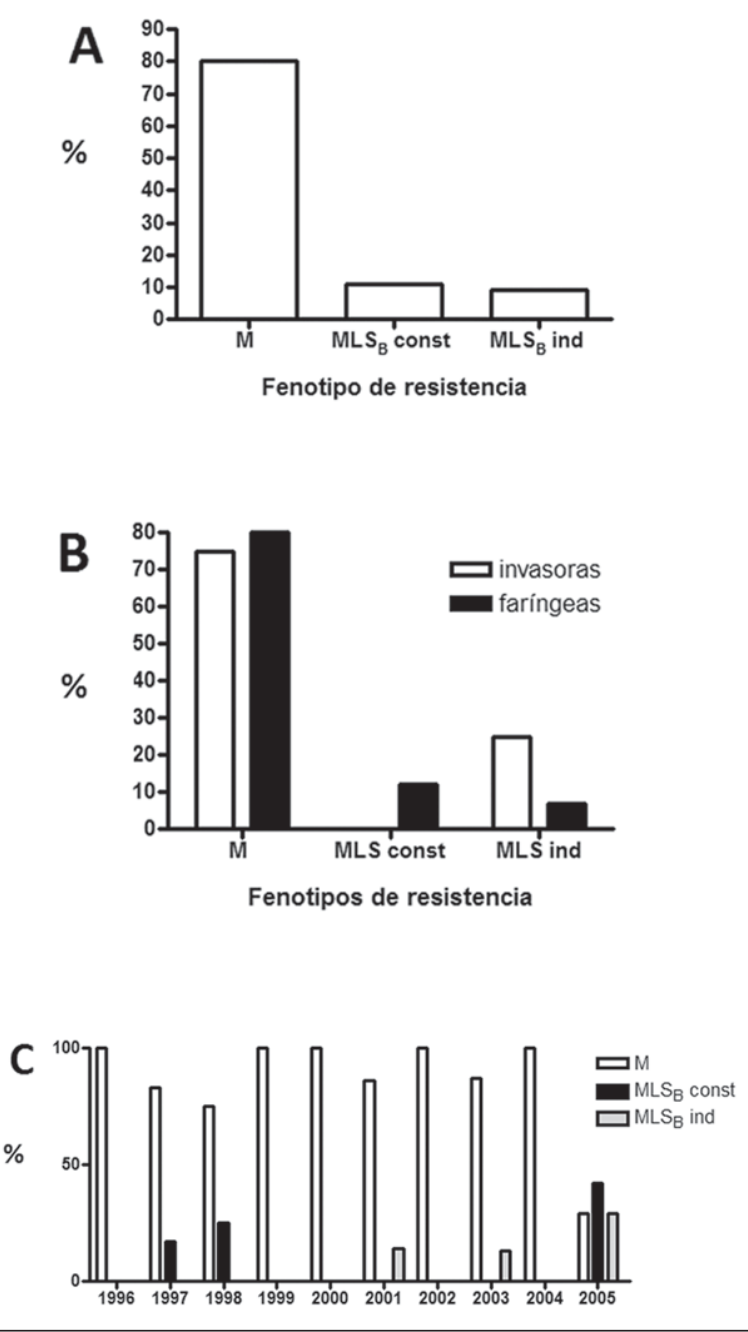

Figura 1. Distribución de fenotipos de resistencia entre las cepas resistentes estudiadas ( $n=45$ ) (A); Distribución de fenotipos de resistencia entre las cepas invasoras y faríngeas (B) y distribución anual de los fenotipos de resistencia (C). de $0,7 \%$ a clindamicina (Tabla 2). El análisis estadístico de estos datos muestra que la distribución de resistencia fue homogénea entre cepas faríngeas e invasoras y que no hubo correlación significativa entre invasividad y resistencia a eritromicina $(p=0,787)$ o resistencia a clindamicina $(p=0,549)$.

La distribución de fenotipos de resistencia entre las cepas resistentes mostró que el fenotipo predominante fue el fenotipo $\mathrm{M}(80 \%)$, seguido por el fenotipo $\mathrm{MLS}_{\mathrm{B}}$ const (11\%) y por último el $\mathrm{MLS}_{\mathrm{B}}$ ind (9\%) (Figura 1A). Se observó que el fenotipo $\mathrm{M}$ fue el predominante tanto en cepas invasoras como faríngeas (Figura 1B). Por otro lado, el fenotipo MLS $_{\mathrm{B}-}$ const se encontró asociado solamente a cepas faríngeas (Figura 1B).

La distribución anual de fenotipos de resistencia en el período de estudio muestra que el primer fenotipo de resistencia que surge fue el M. Luego apareció el fenotipo MLS $_{\mathrm{B}}$ const (1997) y recién en el año 2001 apareció el fenotipo MLS ind (Figura 1C). También se observa que en el año 2005 hubo una distribución homogénea de los 3 fenotipos de resistencia.

\section{Determinación genotípica de resistencia}

La Tabla 3 muestra el fenotipo de resistencia determinado por difusión en agar, el genotipo y su gen asociado determinado por PCR para las 45 cepas resistentes. De estas cepas, 41 de ellas poseían el gen de resistencia concordante al fenotipo (91\%). Tres de ellas $(6,7 \%)$ poseían genes de resistencia que no concordaban con el fenotipo. En una cepa $(2,3 \%)$ no fue detectado ningún gen de resistencia (cepas marcadas con ${ }^{\star}$ en la Tabla 3). El $82,2 \%$ (37/45) de las cepas resistentes poseían el gen mef, el 6,7\% (3/45) de las cepas poseían el gen ermA y el 8,9\% (4/45) de las cepas poseían el gen ermB (Tabla 3). De las tres cepas no concordantes, dos de ellas mostraban el fenotipo $\mathrm{MLS}_{\mathrm{B}}$ const pero poseían solamente el gen mef, mientras que otra cepa que mostraba el fenotipo MLS ${ }_{B}$ ind poseía solamente el gen ermB. Las 4 cepas no concordantes fueron analizadas mediante amplificación del rRNA 16S corroborando la integridad del ADN bacteriano extraído (datos no mostrados). 
Tabla 3. Fenotipo, genotipo y genes de resistencia asociados de las cepas resistentes

\begin{tabular}{|lll|}
\hline Fenotipo & $\begin{array}{l}\text { Genotipo/gen } \\
\text { detectado }\end{array}$ & \% de cepas (n) \\
\hline M & $\mathrm{M} /$ mef & $78 \%(35 / 45)$ \\
& $-/$-* $^{*}$ & $2,2 \%(1 / 45)$ \\
MLSB ind & $\mathrm{MLS}_{\mathrm{B}}$ ind / ermA & $6,7 \%(3 / 45)$ \\
& $\mathrm{MLS}_{B}$ const / ermB* & $2,2 \%(1 / 45)$ \\
MLSB const & $\mathrm{MLS}_{B}$ const / ermB & $6,7 \%(3 / 45)$ \\
& $M /$ mef* $^{*}$ & $4,4 \%(2 / 45)$ \\
\hline
\end{tabular}

*Cepas no concordantes en las cuales el fenotipo no coincide con el genotipo.

\section{Discusión}

Este es el primer estudio de resistencia a eritromicina en $S$. pyogenes con más de 1.000 cepas aisladas de pacientes en Chile, que abarca un período reciente de 10 años y donde todas las cepas resistentes fueron confirmadas genotípicamente.

El estudio de resistencia en cepas de S. pyogenes entre los años 1996 y 2005 confirma que existe $100 \%$ de sensibilidad a penicilina, lo que corrobora que este antibiótico sea el tratamiento antimicrobiano de elección. Respecto a la resistencia a eritromicina y clindamicina, los porcentajes globales de resistencia son similares a los registrados por Palavecino y cols entre 1990 y 1998, quienes describieron 5,4\% de resistencia a eritromicina y $0,7 \%$ de resistencia a clindamicina ${ }^{26}$. Adicionalmente, Camponovo y cols registraron un porcentaje de resistencia a eritromicina y a clindamicina de $13,5 \%$ y $1,4 \%$, respectivamente, entre los años 1995 y 1997 (comunicado en el XV Congreso Chileno de Infectología, 1998), siendo los porcentajes de resistencia a dichos antibióticos más altos registrados en Chile. Coincidentemente, en nuestro trabajo, en esos mismos años, hemos registrado los mayores porcentajes de resistencia a eritromicina. Nuestros datos muestran que a partir del año 1999 los niveles de resistencia disminuyeron en Chile. Esto podría estar relacionado con el hecho de que en 1999 el Ministerio de Salud de Chile (MINSAL) implementó una medida regulatoria que restringe la venta de antibióticos (Ordinario 4C/5015 del 30.09.1999). Esta medida ha tenido un impacto significativo al disminuir el uso indiscriminado de antibióticos, incluyendo el grupo de los macrólidos ${ }^{32}$. Los resultados obtenidos en nuestro trabajo contrastan con valores registrados en otros países. Pérez-Trallero y cols reportaron una resistencia a eritromicina de 21,3\% en España ${ }^{33}$, en Italia la resistencia a eritromicina entre 2001 y 2006 alcanzó el 22,6\% ${ }^{34}$ y en Estados Unidos de Norteamérica llegó a 6,8\% entre 2002 y $2003^{28}$. Estas diferencias pueden deberse a las distintas tendencias en el consumo de antibióticos con respecto a Chile. Hay estudios que demuestran la correlación existente entre consumo de antibióticos y los niveles de resistencia ${ }^{16}$, siendo los antibióticos de acción prolongada como azitromicina los más determinantes en la selección de cepas resistentes, debido a que tienen una ventana selectiva más prolongada ${ }^{35}$. Más aun, en Italia se ha reportado un posible vínculo entre la desaparición de resistencia a macrólidos y un consumo controlado de macrólidos de acción prolongada ${ }^{36}$. Recientemente, Bavestrello y cols ${ }^{37}$ describieron que el consumo de claritromicina ha aumentado en $108 \%$ mientras que el de azitromicina lo ha hecho sólo en $31 \%$ entre 2000 y 2005 en Chile. Sin embargo, el consumo de eritromicina ha disminuido en $72 \%$, por lo que el consumo global de antibióticos ha aumentado solamente en 7,6\% en ese período. El leve aumento del consumo de azitromicina, sumado al bajo crecimiento del consumo global de macrólidos, podrían explicar los bajos niveles de resistencia registrados en Chile en comparación con países europeos.

El fenotipo $\mathrm{M}$ de resistencia es el predominante en Chile, tanto en cepas invasoras como faríngeas (80\%). Sin embargo, se observa que ha disminuido respecto a lo reportado por Palavecino y cols en años anteriores $(87 \%)$. Esto continúa siendo notoriamente diferente a lo que sucede en países como China, en donde existe una clara predominancia del fenotipo $\mathrm{MLS}_{\mathrm{B}}$ const entre las cepas resistentes a eritromicina $(98,8 \%)^{38}$. También en Europa, entre 2005 y 2006 se describe una predominancia del fenotipo $\mathrm{MLS}_{\mathrm{B}}$ const que da cuenta de 58,9\% de las cepas resistentes a macrólidos ${ }^{39}$. De acuerdo a nuestros resultados, los porcentajes de los fenotipos MLS $_{\mathrm{B}}$ ind y MLS $\mathrm{B}_{\mathrm{B}}$ const han aumentado respecto a lo reportado por Palavecino ${ }^{26}$ de $6,25 \%$ a $8,9 \%$ y de $6,25 \%$ a $11,1 \%$, respectivamente.

La distribución homogénea de fenotipos de resistencia en el año 2005 sugiere que los genes de resistencia se van adquiriendo secuencialmente. $\mathrm{Al}$ respecto, cabe destacar que en el año 2006 fueron 
aisladas 3 cepas que poseen dos genes de resistencia cada una: ermB y mef (datos no mostrados). Estos resultados resaltan la necesidad de realizar un seguimiento de la evolución de la resistencia estudiando cepas aisladas en años más recientes.

Es probable que la cepa que presenta un fenotipo $\mathrm{M}$ pero que no presenta ninguno de los genes buscados, posea otro mecanismo de resistencia como, por ejemplo, mutaciones en las proteínas ribosomales que participan en la unión al antibiótico como la proteína L4. Un mecanismo como éste fue descrito en aislados clínicos de Francia por Bingen y cols ${ }^{40}$. Las cepas no concordantes continúan en estudio para la dilucidación de su mecanismo de resistencia.

Los resultados obtenidos permiten concluir que los niveles de resistencia a eritromicina en aislados clínicos de $S$. pyogenes han disminuido significativamente en los últimos años en Chile. La situación en Chile es notoriamente diferente a la de otros países en cuanto a los porcentajes de resistencia y a los fenotipos predominantes. Por otro lado, se vio que en la mayoría de los casos el fenotipo de resistencia coincide con el genotipo determinado por PCR.

\section{Referencias}

1. Carapetis JR, McDonald M, Wilson NJ. Acute rheumatic fever. Lancet 2005; 366 (9480): 155-68.

2. Vucicevic Z, Jajic-Bencic I, Kruslin B, Degoricija V. Toxic shock syndrome due to group A streptococcal pharyngitis and bacteremia in adults. J Microbiol Immunol Infect 2009; 42 (3): 276-9.

3. Simonart T. Group a beta-haemolytic streptococcal necrotising fasciitis: early diagnosis and clinical features. Dermatology 2004; 208 (1): 5-9.

4. Nisbet M, Ansell G, Lang S, Taylor S, Dzendrowskyj P, Holland D. Necrotizing Fasciitis: review of 82 cases in South Auckland. Intern Med J 2010; ahead of print.

5. Baxter F, McChesney J. Severe group A streptococcal infection and streptococcal toxic shock syndrome. Can J Anaesth 2000; 47 (11): 1129-40.

6. Efstratiou A. Group A streptococci in the 1990s. J Antimicrob Chemother 2000; 45 Suppl: 3-12.

7. Schellekens JF, Schouls LM, van Pelt W, Esveld M, van Leeuwen WJ. Group A streptococci: a change in virulence? Neth J Med 1998; 52 (6): 209-17.

8. Efstratiou A, George RC, Tanna A, Hookey JV, Caugant D, Holm SE, et al. Characterisation of group A strepto- cocci from necrotising fasciitis cases in Gloucestershire, United Kingdom. Adv Exp Med Biol 1997; 418: 91-39.

9. Cartwright K, Logan M, McNulty C, Harrison S, George $\mathrm{R}$, Efstratiou A, et al. A cluster of cases of streptococcal necrotizing fasciitis in Gloucestershire. Epidemiol Infect 1995; 115 (3): 387-97.

10. Berríos X, Lagomarsino E, Solar E, Sandoval G, Guzmán B, Riedel I. Post-streptococcal acute glomerulonephritis in Chile--20 years of experience. Pediatr Nephrol 2004; 19 (3): 306-12.

11. Markowitz M. Changing epidemiology of group A streptococcal infections. Pediatr Infect Dis J 1994; 13 (6): 557-60.

12. Mandell G, Bennet J, Douglas R. Principles and practices of Infectious diseases. 6th Ed. Philadelfia: Elsevier. Churchil Livingstone, 2005.

13. Lipsitch M, Samore M. Antimicrobial use and antimicrobial resistance: a population perspective. Emerg Infect Dis 2002; 8: 347-54.

14. Pihlajamaki M, Kotilainen P, Kaurila T, Klaukka T, Palva E, Houvinen P. Macrolide resistant Streptococcus pneumoniae and use of antimicrobial agents. Clin Infect Dis 2001; 33: 483-8.

15. Seppala H, Klaukka T, Vuopio-Varkila J, Muotiala A, Helenius H, Lager K. The effects of changes in the consumption of macrolide antibiotics on eritromycin resistance in group A streptococci in Finland. N Eng J Med 1997; 337: 441-6.

16. Albrich W, Monnet L, Harbarth S. Antibiotic selection pressure and resistance in Streptococcus pneumoniae and Streptococcus pyogenes. Emerg Infect Dis 2004; 10 (3): 514-7.

17. Weisblum B. Erythromycin resistance by ribosome modification. Antimicrob Agents Chemother 1995; 39 (3): 577-85.

18. Leclercq R, Courvalin P. Resistance to macrolides and related antibiotics in Streptococcus pneumoniae. Antimicrob Agents Chemother 2002; 46 (9): 2727-34.

19. Horinouchi S, Byeon WH, Weisblum B. A complex attenuator regulates inducible resistance to macrolides, lincosamides, and streptogramin type B antibiotics in Streptococcus sanguis. J Bacteriol 1983; 154 (3): 1252-62.

20. Seppala H, Skurnik M, Soini H, Roberts MC, Huovinen P. A novel erythromycin resistance methylase gene (ermTR) in Streptococcus pyogenes. Antimicrob Agents Chemother 1998; 42 (2): 257-62.

21. Weisblum B. Insights into erythromycin action from studies of its activity as inducer of resistance. Antimicrob Agents Chemother 1995; 39 (4): 797-805.

22. Clancy J, Petitpas J, Dib-Hajj F, Yuan W, Cronan M, Kamath $\mathrm{AV}$, et al. Molecular cloning and functional analysis 
of a novel macrolide-resistance determinant, mefA, from Streptococcus pyogenes. Mol Microbiol 1996; 22 (5): 86779.

23. Sutcliffe J, Tait-Kamradt A, Wondrack L. Streptococcus pneumoniae and Streptococcus pyogenes resistant to macrolides but sensitive to clindamycin: a common resistance pattern mediated by an efflux system. Antimicrob Agents Chemother 1996; 40 (8): 1817-24.

24. Maggi L, Alvarez F, Guerra C, Prado V, Berríos X. Vigilancia de la sensibilidad a antimicrobianos de Streptococus beta-hemolíticos aislados entre 1982 y 1987. Rev Chil Infect 1989; 6: 23-7.

25. Berrios X, Bedregal P, Ramírez G, Riedel I. Infección por estreptococo grupo A invasor, caracterización bacteriológica. Rev Chil Infect 1994; 11: 26-34.

26. Palavecino EL, Riedel I, Berrios X, Bajaksouzian S, Johnson D, Kaplan E, Jacobs MR. Prevalence and mechanisms of macrolide resistance in Streptococcus pyogenes in Santiago, Chile. Antimicrob Agents Chemother 2001; 45 (1): 339-41.

27. Clinical and Laboratory Standards Institute (CLSI). Methods for dilution antimicrobial susceptibility tests for bacteria that grow aerobically.: Wayne, P.A., 2006.

28. Richter SS, Heilmann KP, Beekmann SE Miller NJ, Miller AL, Rice CL, et al. Macrolide-resistant Streptococcus pyogenes in the United States, 2002-2003. Clin Infect Dis 2005; 41 (5): 599-608.

29. Bingen E, Fitoussi F, Doit C, et al. Resistance to macrolides in Streptococcus pyogenes in France in pediatric patients. Antimicrob Agents Chemother 2000; 44 (6): 1453-7.

30. Kim H, Fay M, Feuer E, Midthune D. Permutation tests for joinpoint regression with applications to cancer rates. Stat Med 2000; 19: 335-51.

31. R Development Core Team. R Foundation for Statistical Computing A language and environment for statistical computing. 2009. Vienna, Austria. ISBN 3-90005107-0, URL http://www.R-project.org. (Revisado el $15 / 06 / 2010)$.
32. Bavestrello L, Cabello A, Casanova D. [Impact of regulatory measures in the trends of community consumption of antibiotics in Chile]. Rev Med Chile 2002; 130 (11): 1265-72.

33. Pérez-Trallero E, Marimon JM, Ercibengoa M, Giménez MJ, Coronel P, Aguilar L. Antimicrobial susceptibilities of amoxycillin-non-susceptible and susceptible isolates among penicillin-non-susceptible Streptococcus pneumoniae. Clin Microbiol Infect 2007; 13 (9): 937-40.

34. Montagnani F, Stolzuoli L, Croci L, Rizzuti C, Arena F, Zanchi A, et al. Erythromycin resistance in Streptococcus pyogenes and macrolide consumption in a central Italian region. Infection 2009; 37 (4): 353-7.

35. Cizman M. The use and resistance to antibiotics in the community. Int J Antimicrob Agents 2003; 21 (4): 297 307.

36. De Rosa R, Avolio M, Stano P, Modolo ML, Camporese A. [Disappearance of Streptococcus pyogenes macrolide resistance in an area of northeastern Italy: a possible link with rational long-acting macrolide consumption]. Infez Med 2009; 17 (2): 82-7.

37. Bavestello L, Cabello A. Consumo comunitario de antibióticos en Chile 2000-2008. Rev Chil Infectol 2010; 27, en prensa.

38. Liu X, Shen X, Chang H, Huang G, Fu Z, Zheng Y, et al. High macrolide resistance in Streptococcus pyogenes strains isolated from children with pharyngitis in China. Pediatr Pulmonol 2009; 44 (5): 436-41.

39. Gracia M, Díaz C, Coronel P, Gimeno M, García-Rodas $\mathrm{R}$, Rodríguez-Cerrato V, et al. Antimicrobial susceptibility of Streptococcus pyogenes in Central, Eastern, and Baltic European Countries, 2005 to 2006: the cefditoren surveillance program. Diagn Microbiol Infect Dis 2009; 64 (1): 52-6.

40. Bingen E, Leclercq R, Fitoussi F, Brahimi N, Malbruny B, Deforche D, et al. Emergence of group A Streptococcus strains with different mechanisms of macrolide resistance. Antimicrob Agents Chemother 2002; 46 (5): 1199-203. 